\title{
Non-destructive investigation of fibronectin adsorption on titanium surfaces using PM-RAIRS: effect of surface hydroxylation
}

\author{
Stéphanie Boudesocque, ${ }^{a}$ Olivier Dargaud,${ }^{a}$ Lorenzo Stievano, ${ }^{a}{ }^{*}$ Christophe Méthivier ${ }^{\mathrm{a}}$, \\ Jean-François Lambert, ${ }^{a}$ Thibaud Coradin $^{b}$ and Claire-Marie Pradier ${ }^{a}$
}

${ }^{a}$ Laboratoire de Réactivité de Surface - UMR CNRS 7197, Université Pierre et Marie Curie - Paris 6, Case 178, 4 place Jussieu, 75252 Paris Cedex 05, France.

${ }^{\mathrm{b}}$ Laboratoire de Chimie de la Matière Condensée - UMR CNRS 7574, Université Pierre et Marie Curie - Paris 6, 75252 Paris CEDEX 05,

\begin{abstract}
PM-RAIRS is a useful non-destructive technique to study semi-quantitatively the adsorption of proteins on metal surfaces. In this work, we employed PM-RAIRS to study the effect of the degree of hydroxylation of the surface of titanium metal foils, obtained by specific acidic or basic treatments, on the adsorption of fibronectin, a model plasma adhesion protein. Our results indicate that the degree of hydroxylation does not significantly influence the adhesion properties of fibronectin, which is one of the main steps in titanium osseointegration process.
\end{abstract}

Keywords: Titanium; Biocompatibility; Fibronectin; Adsorption, PM-RAIRS.

Running title: PM-RAIRS investigation of fibronectin adsorption on titanium surfaces

\footnotetext{
* Corresponding author: e-mail: lorenzo.stievano@ upmc.fr, fax: +33-144276033, tel.: +33-144275519.
} 


\section{Introduction}

Titanium metal has been used as a base material for bone implant since 1960. The first documented account of the ability of titanium to build a structural and functional connection between its surface and the living bone was provided by Brånemark, who observed that titanium can successfully fuse into bone when osteoblasts grow on and into its rough surface. This property, named osseointegration and connected to the unusual surface properties of titanium in a biological medium, has largely contributed to the science of medical bone, dentistry and joint replacement techniques. $^{[1-3]}$

A large number of studies on titanium osseointegration have been carried out both in vivo ${ }^{[4-6]}$ and in vitro ${ }^{[7-10]}$. These studies pointed out that the success of implant integration relies on the formation of a bioactive interface between the metal and the body fluid. One of the main steps in this process consists in the adsorption, on the oxidised/functionalised metal surface, of adhesion plasma glycoproteins, such as fibronectin and vitronectin, which become activated by a change in their configuration upon adsorption. ${ }^{[1,12]}$ The following adhesion of osteoblastic cells occurs through specific receptors of the cell membrane known as integrins, which recognize particular sequences of amino acids available only if the adhesion proteins are in their activated state. ${ }^{[13]}$

However, the detailed mechanism of adsorption and the configuration of these proteins adsorbed on titanium surfaces are not yet completely understood in terms of surface chemistry at the molecular level, even though this knowledge is essential to elucidate the origin of the unique properties of integration of titanium in biotic environments. Indeed, in the literature, several studies are devoted to the adsorption of fibronectin, mainly on well-defined titanium oxide powders ${ }^{[14-16]}$ as model systems for real titanium metal surfaces.

In fact, the native surface of titanium metal is usually covered with a passivation oxide layer with a thickness of a few nm. In order to improve its implant properties, titanium metal can be pretreated, in order to modulate both its macroscopic structure (rugosity) and its surface composition. Both parameters are in fact important in the performance of the implant:

1 On the one hand, it is nowadays well-established that a rugosity between 1 to $10 \mu \mathrm{m}$ favours osseointegration. ${ }^{[17]}$

2 On the other hand, different chemical treatments, such as alkali chlorides solutions, ${ }^{[18]}$ acidic $^{[19]}$ or basic conditions, ${ }^{[20]}$ are known to influence the adhesion of cells. However, the most effective treatments usually combine a corrosive treatment with an oxidative one. ${ }^{[21]}$

However, most of the previously-reported chemical treatments not only affect the chemical composition of the interface, but may also strongly change the rugosity and the composition of the surface layer, so that it is often very difficult to distinguish between the relative effects of these parameters on the protein adsorption. ${ }^{[22]}$ 
In order to improve the understanding of the adsorption of fibronectin on titanium surfaces, and in particular to shed more light on the influence of the chemical treatment on the adsorptive properties of the protein, we have started a combined spectroscopic study by XPS and infrared spectroscopy in the reflection absorption mode with polarisation modulation of the incident beam (PM-RAIRS). This surface-dedicated technique, pertinent only to plane metal sheets with rugosity below $1 \mu \mathrm{m}$, is very sensitive to the nature and the orientation of the adsorbed molecules, allowing their identification and, in some cases, the study of their adsorption geometry with respect to the surface. In this communication, we present the results on the adsorption of fibronectin on titanium surfaces having very similar rugosities, the same composition but a different degree of hydroxylation.

\section{Materials and Methods}

Prior to use, high purity titanium sheets (Goodfellow, purity 99.99\%, 10 × $10 \times 1 \mathrm{~mm}$ ) were mechanically polished down to $1 / 4 \mu \mathrm{m}$ using suspensions of diamond particles of decreasing sizes, washed with MilliQ water and acetone in an ultrasound bath, and finally dried in flowing Ar.

The titanium sheets were put in contact for $20 \mathrm{~min}$ with bovine plasma fibronectin (FN, Calbiochem, PBS buffer) solutions of increasing concentrations after one of the following pretreatments:

1. Simple polishing, washing and drying;

2. A treatment of $20 \mathrm{~min}$ in a piranha solution, i.e., a mixture of concentrated $\mathrm{H}_{2} \mathrm{SO}_{4}$ and an aqueous solution of $30 \% \mathrm{H}_{2} \mathrm{O}_{2}$. The samples are then washed and dried as described above. This treatment produces highly hydroxylated surfaces (see supplementary data).

3. A treatment of $20 \mathrm{~min}$ in a $5 \mathrm{M} \mathrm{NH}_{3}$ aqueous solution. The samples are then washed and dried as described above. This treatment is expected to provide thick oxide layers with low hydroxylation (see supplementary data).

A contact time of 20 min was sufficient to reach adsorption equilibrium, in agreement with previous studies of the dynamics of fibronectin adsorption on titanium oxide surfaces. ${ }^{[23]}$ The surface of the metal sheets was then washed 10 times with a PBS solution and 5 times with MilliQ water in order to remove the solution covering the surface and the co-adsorbed phosphate.

AFM analyses were performed with a Caliber AFM microscope (VEECO Instruments Inc.) after polishing and after the different chemical treatments preceding protein adsorption.

XPS analyses of the metal sheets before protein adsorption were performed on a Phoibos 100 $\mathrm{X}$-ray photoelectron spectrometer (SPECS GmbH, Germany) with the $\mathrm{Mg} \mathrm{K \alpha} \mathrm{X}$-ray source (hv = $1253.6 \mathrm{eV})$. Spectra were recorded with $20 \mathrm{eV}$ pass energy for the survey scan and with $10 \mathrm{eV}$ pass energy for the $\mathrm{C} 1 \mathrm{~s}, \mathrm{O} 1 \mathrm{~s}, \mathrm{~N}$ 1s, and Ti 2p regions. High resolution XPS conditions have been fixed: 
'fixed analyser transmission' analysis mode, a $7 \times 20 \mathrm{~mm}$ entrance slit and $150 \mathrm{~W}$ electron beam power. A takeoff angle of $90^{\circ}$ from the surface was employed. Element peak intensities were corrected by Scofield factors, ${ }^{[24]}$ the spectra were fitted using the Casa XPS v.2.3.13 Software (Casa software Ltd. UK) and by applying a Gaussian/Lorentzian ratio, G/L equal to 70/30.

PM-RAIRS spectra were recorded with a FT-IR instrument (Nicolet 5700 spectrometer) at a 4 $\mathrm{cm}^{-1}$ resolution, the reflected light being focused on a nitrogen-cooled mercury cadmium telluride (MCT) detector. A ZnSe grid polariser and a ZnSe photoelastic modulator to modulate the incident beam between $\mathrm{p}$ and s polarizations (HINDS Instruments, PEM90, modulation frequency $=36 \mathrm{kHz}$ ) are placed prior to the sample. The use of polarisation modulation allows one to perform rapid analyses of the samples after immersion without purging the atmosphere, nor requiring a reference spectrum of the bare support.

\section{Results and Discussion}

\section{XPS-AFM Surface Characterisation}

The morphology of the surface of the titanium sheets was examined by AFM (Caliber AFM microscope, VEECO Instruments Inc.) after polishing and different chemical treatments preceding protein adsorption, always shows flat surfaces with no apparent rugosity down to $0.1 \mu \mathrm{m}$ (not shown).

Survey XPS spectra (not shown) indicate the absence of contaminants since no peaks attributable to elements other than the expected ones (Ti, O, C, N, and P) were visible in the spectra. High resolution spectra of the Ti $2 p$ region (not shown) indicate the presence of tetravalent titanium in all studied samples, whereas no signals belonging to Ti in lower oxidation states is present. This result is in line with the complete coverage of studied metal sheets with homogeneous $\mathrm{TiO}_{2}$ surface layers of at least a few nm.

High resolution XPS spectra of the Ols region were measured to evaluate of the degree of hydroxylation of the oxide surface layer of the metal sheets (Fig. 1). These spectra can be fitted using three spectral contributions:

1. $530.1 \pm 0.2 \mathrm{eV}$, attributed to oxide in $\mathrm{TiO}_{2}[1]$.

2. $531.7 \pm 0.2 \mathrm{eV}$, attributed to surface hydroxyls.

3. $533.0 \pm 0.2 \mathrm{eV}$, attributed to adsorbed water molecules.

The results of these fits shows that the untreated sample is the less hydroxylated one, with a ratio hydroxyls/oxide of 0.25 ; the sample treated in ammonia solutions is slightly more hydroxylated 
(ratio hydroxyls/oxide of 0.30), whereas the sample treated in the piranha solution has a significantly higher surface hydroxylation (ratio hydroxyls/oxide of 0.63 ).

\section{Fibronectin Adsorption}

When studying surface-adsorbed proteins using IR techniques, the spectral regions of interest includes the amide I and amide II bands, characteristic of the protein and in part indicative of their configuration. The amide I band occurs between 1600 and $1700 \mathrm{~cm}^{-1}$ and consists for $80 \%$ in the $\mathrm{C}=\mathrm{O}$ stretching vibration of the amide group. ${ }^{[25,26]}$ The amide II band contains $60 \% \mathrm{~N}-\mathrm{H}$ bending coupled to some $\mathrm{C}-\mathrm{N}$ stretching $(40 \%)$ of the amide group. ${ }^{[23,25]}$ Figure 2 shows that, with an increase in FN concentration, the overall intensity of the amide bands (I and II) also increases, indicating progressively greater surface concentrations of FN. In principle, information on the configuration of the protein chain can sometimes be deduced from variations in the band position and/or the amide I / amide II intensity ratio; these two parameters remain unchanged along the series of measurements corresponding to increasing protein loadings, suggesting that the configuration of adsorbed fibronectin molecules is independent of coverage.

Figure 3 presents the adsorption isotherms of FN on the titanium sheets as a function of the protein concentration, as estimated from the average intensity of the amide I band (average taken on triplicate series of measurements). The measured isotherms present the same shape for all three series of titanium sheets studied, irrespective of the chemical treatment used; in particular, the saturation of the surface is obtained for the same protein concentration $\left(0.15 \mathrm{mg} \cdot \mathrm{mL}^{-1}\right)$, which corresponds to about half of the natural fibronectin concentration in human plasma. This would indicate that the affinity of the protein for the surface is not dependent on the surface hydroxylation obtained after the different treatments. Moreover, the vertical position of the adsorption plateau does not vary much following the two studied treatments, suggesting that they do not affect significantly the saturation coverage.

These considerations can be put on a more quantitative basis if the adsorption obeys a Langmuir model, whose linearized form is:

$$
\frac{c}{A}=\frac{1}{B_{a d s} A_{\max }}+\frac{c}{A_{\max }}
$$

where $A$ is the area of the amide I band, $A_{\max }$ is the area of the amide I band at the maximum of the protein surface concentration, $c$ is the equilibrium concentration, and $B_{a d s}\left(\mathrm{~mL} \mathrm{mg}^{-1}\right)$ is the adsorption coefficient which measures the affinity of the protein toward adsorption to the metal surface. A plot of $c / A$ vs $c$ indeed shows a linear trend for all samples (Fig. 5), indicating that the Langmuir hypotheses are satisfied. The untreated sheets as well as those treated in $\mathrm{NH}_{3}$ and in $\mathrm{H}_{2} \mathrm{SO}_{4} / \mathrm{H}_{2} \mathrm{O}_{2}$ solution present only slightly different $B_{a d s}$ values (14(1), 17(2) and 20(2) $\mathrm{mL} \mathrm{mg}^{-1}$, 
respectively): even though the two treatments may induce a slight increase of the affinity of the surface for fibronectin, the fact that they produce a very different degree of surface hydroxylation has virtually no effect on the protein affinity for the surface.

In order to validate our approach and to verify that the intensity of the amide bands in the PMRAIRS spectra is proportional to the amount of adsorbed protein, we followed the attenuation of the high resolution XPS Ti2 $p$ spectrum (expressed as $-\ln \left(\mathrm{I} / \mathrm{I}_{0}\right)$, where $\mathrm{I}$ is the relative intensity of the Ti2 $2 p$ XPS peak, and $I_{0}$ is the intensity of the same peak without adsorbed fibronectin), which is proportional to the adsorbed amount on increasing protein concentration using the same samples immediately after the measurement of the PM-RAIRS spectra. Figure 4 presents a comparison between the attenuation of the XPS Ti $2 p$ peak on the one hand, and the intensity of the amide I band in the PM-RAIRS spectra on the other hand, for the simply polished titanium sheets. One can see that the two graphs are virtually superimposable apart from an arbitrary normalization constant, indicating a full agreement between XPS and PM-RAIRS results.

It is worth noting that such a comparison is only possible because the PM-RAIRS technique is a fast, non-destructive method, in contrast with other techniques such as XPS, which has been so far considered as the reference technique for similar protein adsorption studies. ${ }^{[14,15,22]}$ Therefore, the present results establish the feasibility of quantitative studies of protein adsorption by PMRAIRS.

\section{Conclusions}

PM-RAIRS was successfully employed to study the effect of the degree of hydroxylation of the surface of titanium metal foils, obtained by specific acidic or basic treatments, on the adsorption of fibronectin, a model plasma adhesion protein. Our results indicate that, irrespective of the degree of hydroxylation of the surface, the adsorption of fibronectin can be modelled using the Langmuir model. Moreover, the amount of adsorbed protein does not vary significantly with the degree of hydroxylation of the surface, indicating that the latter does not influence significantly the adhesion properties of fibronectin. These results, which were validated by coupling them with parallel XPS studies on the same materials, are in contradiction with previous studies, ${ }^{[20]}$ which suggested that the specific surface hydroxylation of the titanium oxide surface is one of the key factors governing the specific properties of titanium implant materials. 


\section{Figure Captions}

Fig. 1 High resolution XPS spectra of the Ols region for the samples after the different treatments (from left to right): Polished Ti metal, Ti metal treated in NH3, Ti metal treated in Piranha solution.

Fig. 2 PM-RAIRS spectra of fibronectin on titanium sheets treated in $\mathrm{NH}_{3}$ adsorbed from aqueous solutions with increasing protein concentrations.

Fig. 3 Adsorption isotherms of fibronectin on titanium surfaces as a function of the fibronectin concentration.

Fig. 4 Comparison between FN adsorption as a function of FN concentration onto titanium sheets by XPS and PM-RAIRS measurements

Fig. 5 Langmuir plot of the adsorption isotherms of fibronectin on titanium surfaces for the three series of samples. 

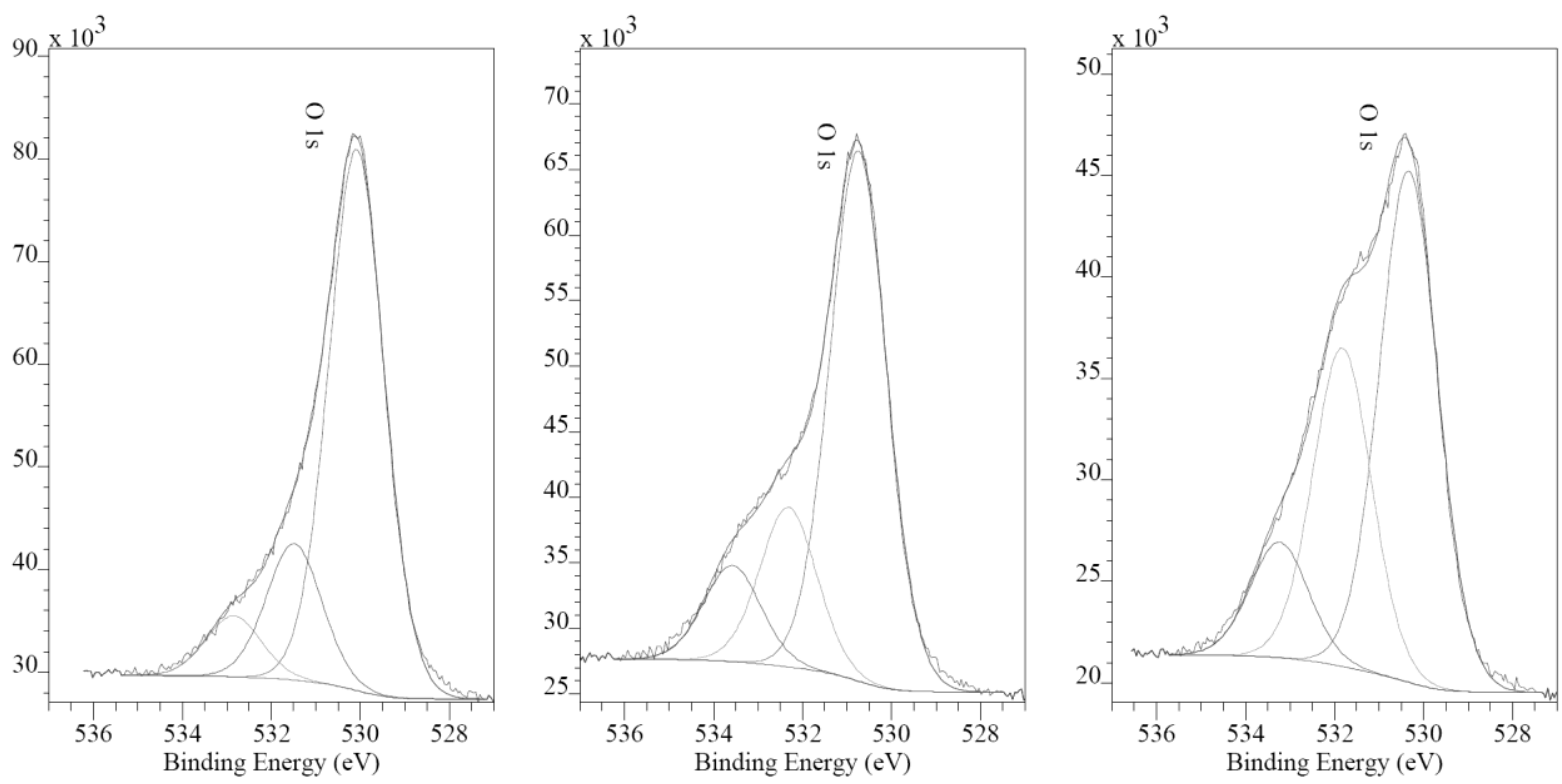

Fig. 1 


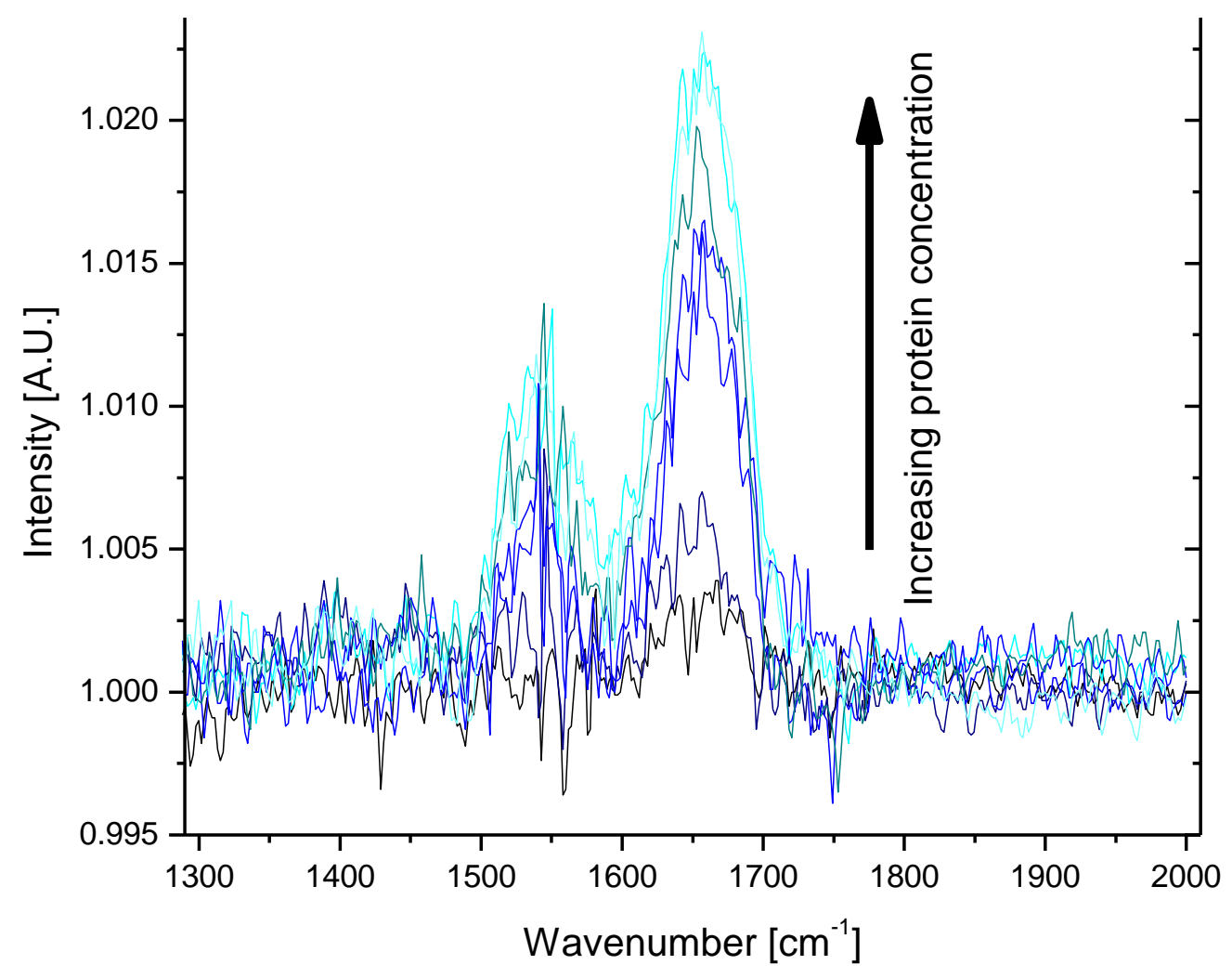

Fig. 2 


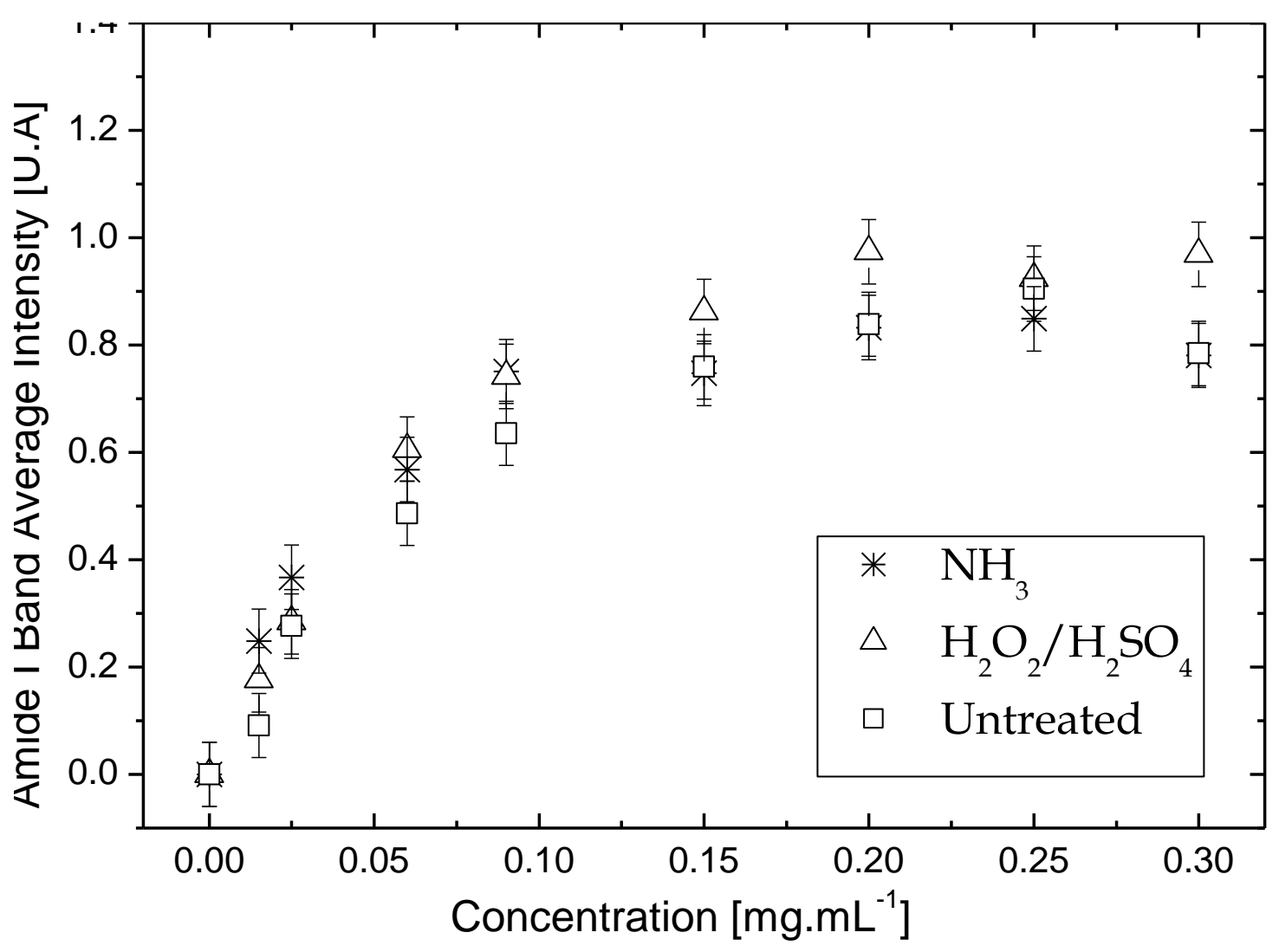

Fig. 3 


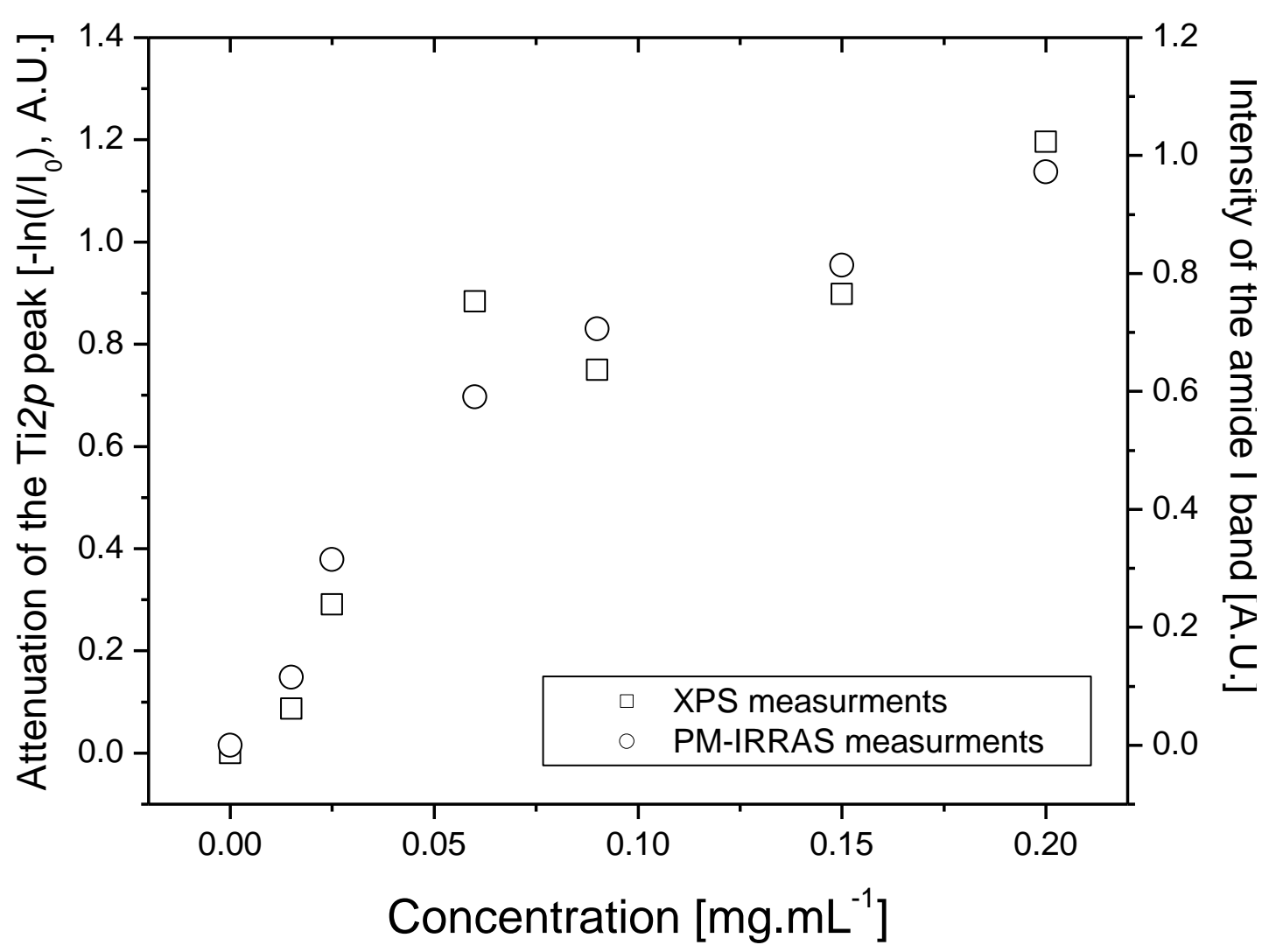

Fig. 4 


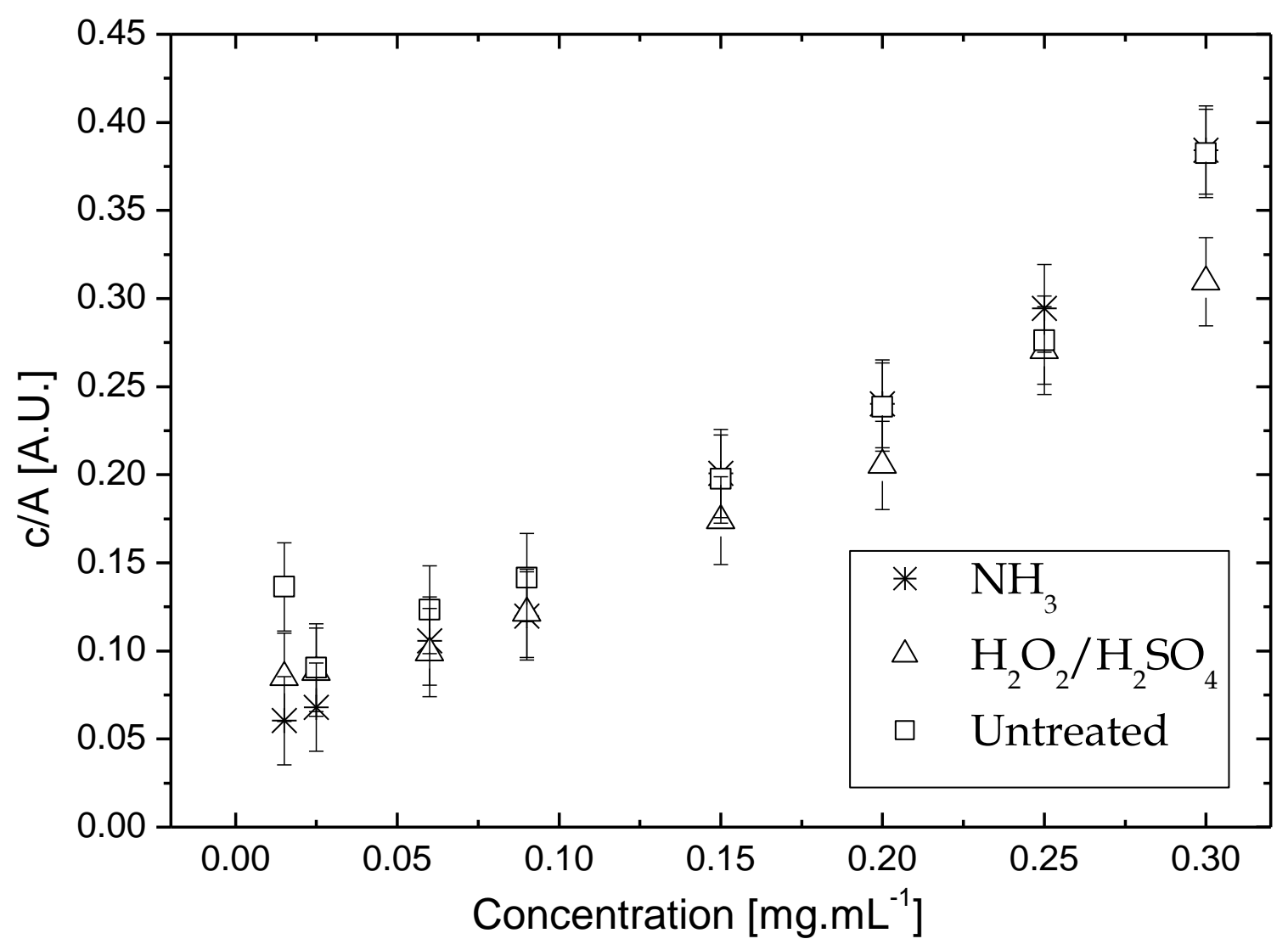

Fig. 5 


\section{References}

[1] L. Linder, A. Carlsson, L. Marsal, L. M. Bjursten, P.-I. Branemark, J. Bone Joint Surgery 1988, 70-B, 550.

[2] T. Albrektsson, P. I. Brånemark, H.-A. Hansson, B. Kasemo, K. Larsson, I. Lundström, D. H. McQueen, R. Skalak, Ann. Biomed. Eng. 1983, V11, 1.

[3] H. Emneus, U. Stenram, J. Baecklund, Acta Orthop. Scand. 1960, 30, 226.

[4] H. J. Ronold, S. P. Lyngstadaas, J. E. Ellingsen, Biomater. 2003, 24, 4559.

[5] H. J. Ronold, J. E. Ellingsen, Biomater. 2002, 23, 4211.

[6] G. Giavaresi, M. Fini, A. Cigada, R. Chiesa, G. Rondelli, L. Rimondini, P. Torricelli, N. N. Aldini, R. Giardino, Biomater. 2003, 24, 1583.

[7] B. S. Park, S. J. Heo, C. S. Kim, J.-E. Oh, J.-M. Kim, G. Lee, W. H. Park, C.-P. Chung, B.M. Min, J. Biomed. Mater. Res. A 2005, 74, 640.

[8] D. D. Deligianni, N. Katsala, S. Ladas, D. Sotiropoulou, J. Amedee, Y. F. Missirlis, Biomater. 2001, 22, 1241.

[9] I. Degasne, M. F. Baslé, V. Demais, G. Huré, M. Lesourd, B. Grolleau, L. Mercier, D. Chappard, Calcif. Tissue Int. 1999, 64, 499.

[10] J. Lincks, B. D. Boyan, C. R. Blanchard, C. H. Lohmann, Y. Liu, D. L. Cochran, D. D. Dean, Z. Schwartz, Biomater. 1998, 19, 2219.

[11] M. Morra, Eur. Cell. Mater. 2006, 12, 1.

[12] H. Schliephake, D. Scharnweber, J. Mater. Chem. 2008, 18, 2404.

[13] B. G. Keselowsky, D. M. Collard, A. J. Garc1a, J. Biomed. Mater. Res. A 2003, 66, 247.

[14] D. E. MacDonald, B. Markovic, A. L. Boskey, P. Somasundaran, Colloids Surf. B: Biointerfaces 1998, 11, 131.

[15] D. E. MacDonald, N. Deo, B. Markovic, M. Stranick, P. Somasundaran, Biomater. 2002, 23, 1269.

[16] M. A. Strehle, P. Rösch, R. Petry, A. Hauck, R. Thull, W. Kiefer, J. Popp, Phys. Chem. Chem. Phys. 200465232

[17] A. Bagno, C. Di Bello, J. Mater. Sci. Mater. Med. 2004, 15, 935.

[18] A. Klinger, D. Steinberg, D. Kohavi, M. N. Sela, J. Biomed. Mater. Res. A 1997, 36, 387.

[19] K. W. Allen, H. S. Alsalim, J. Adhesion 1974, 6, 229.

[20] T. Kokubo, Thermochim. Acta 1996, 280-281, 479.

[21] X.-X. Wang, S. Hayakawa, K. Tsuru, A. Osaka, J. Biomed. Mater. Res. A 2001, 54, 172.

[22] S. R. Sousa, P. Moradas-Ferreria, M. A. Barbosa, J. Mater. Sci. Mater. Med. 2005, 16, 1173.

[23] S. R. Sousa, M. M. Bras, P. Moradas-Ferreira, M. A. Barbosa, Langmuir 2007, 23, 7046.

[24] J. H. Scofield, J. Electron Spectrosc. Relat. Phenom. 1976, 8, 129.

[25] B. H. Stuart, in Infrared Spectroscopy: Fundamentals and Applications (Ed.: B. H. Stuart), John Wiley \& Sons, Ltd, Chichester, West Sussex (England), 2004, pp. 137.

[26] M. J. Desroches, N. Chaudhary, S. Omanovic, Biomacromol. 2007, 8, 2836. 